\title{
Lightweight RFID Privacy Protocol Based on Universal Hash Function
}

\author{
Shu-jing Gao, Rui-quan Zhang \\ College of Information Science and Technology, Qingdao University of Science and Technology, Qingdao, 266061, China
}

\begin{abstract}
Ownership transfer privacy, including forward untraceability and backward untraceability, is a specific privacy problem in supply chain. A lightweight ownership transfer privacy protocol with mutual authentication based on universal hash function is proposed. There are two keys in $\pi_{\text {OTP }}$. The private key that generated by pseudo random number generator is updated on every reply to the request of the reader and ensures the anonymity of the tag. The public key that generated by universal hash function is updated after every successful authenticating and ensure the mutual authentication, correctness and privacy. The security and privacy of the $\pi_{\text {OTP }}$ is ensured by the collision resistance of the universal hash function.
\end{abstract}

Keywords-ownership transfer privacy; universal hash function; pseudo random number generator; RFID.

\section{INTRODUCTION}

In the system of supply-chain based on RFID, the ownership of the tagged item, and the RFID attached with it, will be exchanged between the different entities such as factories, retailers and the customers. At the moment of the transfer, both new and old owners have the same information about the tag. This might cause privacy problems. At that situation, there might be two attacks. (i) The new owner might track the previous interactions of the old owner and the tag or (ii) the old owner might track the previous interactions of the new owner [1]. The aforementioned attacks are called backward traceability and forward traceability, respectively [2][3]. An ownership transfer protocol allows transferring the rights over a tag from the current owner to the new one in a secure and private way.

Kardas etc.[4] taking into account the tradeoff between implementation complexity and security, proposed a ownership transfer privacy protocol based on hash function and PRNG. But the scheme considers only the authentication of reader to tag and suffers from location privacy attack. The security mechanism proposed by Molnar etc.[5] relied on the control of ownership by Trusted Third Party(TTP) to ensure the protection of ownership transfer. In the scheme, the backend database acting as the TTP retains the control right of the tag. The temporary owners and the readers can only obtain part information of the tags.

Another scheme [6] achieves backward privacy under an assumption that the adversary misses one subsequent successful ownership transfer privacy protocol between the reader and the compromised tag. But the scheme did not consider forward privacy problem.

In this paper, a new ownership transfer security protocol $\pi_{\text {OTP }}$ that making use of universal hash function and pseudorandom number generator as security mechanisms is proposed. We prove that $\pi_{\text {OTP }}$ satisfies the attributes of mutual authentication, correctness and ownership transfer privacy.

The outline of the paper is as follows. Section 2 describes the security and privacy model that includes attributes of the system, attack ability of the adversary and the security and privacy objective. In section 3 , the proposed ownership transfer protocol $\pi_{\text {OTP }}$ is described in a detail.

The security and privacy analysis of the $\pi_{\text {OTP }}$ is shown in section 4 . In section 5 , we conclude the paper.

\section{SECURITY AND PRIVACY Model}

The communicating parties are a database, a reader and a tag. The database maintains information for the tags that it owns. In this paper, the term reader is used to mean a combination of a backend database and the reader.

Forward privacy is regarded as the highest privacy level in [7]. In this paper, we consider the even stronger adversary and suppose the tags can be used normally after tampered by the adversary.

The three cryptographic characteristics should be satisfied by the RFID protocol are security, privacy and correctness.

In the RFID context, the security means the illegal tags or readers should not be taken as legitimate ones by the system. The scheme is security if it can provide mutual authentication of both readers and tags.

The privacy means the protocol should not leak any information about the user to the adversary. As has been discussed in the former section, the ownership transfer privacy containing backward untraceability and forward untraceability has the highest privacy level.

Correctness is another attribute that should be satisfied by the scheme. The correctness requires the RFID system to efficiently provide normal service for legitimate readers and tags. In practice, some attacks, such as desynchronizing attack [8], may make inconsistency between the tag and the database cause the tag enter a state of unrecoverable and cannot be identified correctly in subsequent sessions. 


\section{THE PROPOSED PROTOCOL $\pi_{\text {OTP }}$}

The authentication process is composed of 4 steps and is compatible with EPCglobal C1G2. The security components the protocol adopted are universal hash function and pseudorandom number generator.

The notations used in $\pi_{\text {OTP }}$ are as flows.

$T_{i}:$ The tag

$R:$ The reader

$D$ : The backward database

$a_{j}$ : The random number generated by the reader in query step of the $j$ th protocol process

$r_{j}$ : The random number generated by the tag in the $j$ th protocol process

$a_{j}$ : The random number generated by the database in the 3 rd step of tag authenticating reader of the $j$ th protocol process

$\kappa_{0}^{i}:$ The initial key of the $\operatorname{tag} T_{i}$

$\kappa_{j-1}^{i}$ : The key used by the tag $T_{i}$ in the $j$ th authentication

$\kappa_{j}^{i}$ : The key of the $\operatorname{tag} T_{i}$ updated after the $j$ th authentication

The authentication protocol is comprised of 4 steps. The $j$ th protocol process $(j=1,2, \ldots)$ is shown in Fig. 1 . The key of the $T_{i}$ is $\kappa_{j-1}^{i}$ at that time.

Step 1: Query. The reader generates random number $a_{j}$ and sends it to the tag.

Step 2: $\mathrm{R}$ authenticating $\mathrm{T}$.
(1) The tag generates a new pseudorandom number $r_{j}$ when receiving $a_{j}$. After that, it selects hash function $h_{\kappa_{j-1}}$ by $\kappa_{j-1}$ and computes $\quad \mathrm{s}_{1}=h_{\kappa_{j-1}}\left(a_{j} \oplus r_{j} \oplus \kappa_{j-1}\right)|| r_{j} \quad$ and sends the result to the reader. reader computes $\mathrm{s}_{2}=h_{\kappa_{j-1}}^{i}\left(a_{j} \oplus r_{j} \oplus \kappa_{j-1}\right)$ for each tag when receiving $S_{1}$. The authentication of the tag is successful if a match $\mathrm{S}_{1}=S_{2}$ is found.

Step 3: T authenticating R.

(1) The reader selects hash function $h_{\kappa_{j-1}}$ by $\kappa_{j-1}$ and computes $\kappa_{j}=h_{\kappa_{j-1}}\left(a_{j}|| r_{j}\right)$ as the new key.

Next, it generates a new pseudorandom number $a_{j}$ and selects hash function $h_{\kappa_{j}}$ by $\kappa_{j}$. At last, $h_{\kappa_{j}}\left(a_{j} \oplus \kappa_{j-1}\right)$ is computed and $\mathrm{s}_{3}=h_{\kappa_{j}}\left(a_{j}^{\prime} \oplus \kappa_{j-1}\right)|| a_{j}^{\prime}$ is sent to the tag.

The tag executes the same operation as the reader and generates new key after receiving $S_{3}$. If a match is found between the received and the computed value, it means that the reader is legitimate and the authentication of the reader is successful. The key is updated to $\kappa_{j}$ at last.

Step 4: Further operation. After the mutual authentication of the tag and the reader, the hash value of the $\mathrm{EPC} h_{\kappa_{j}}(E P C)$ is sent to the reader for further operation.

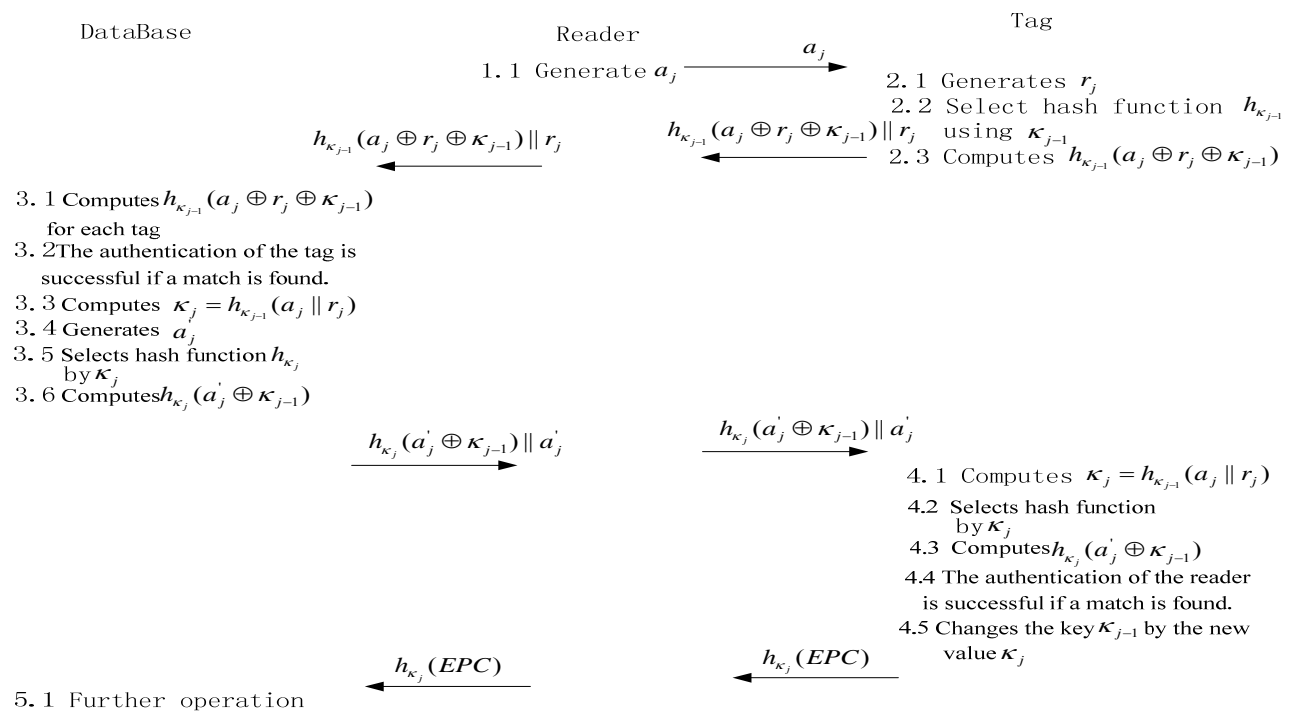

Figure 1. The Process of the Protocol $\pi_{\text {OTP }}$ 


\section{SECURITY AND PRIVACY ANALYSIS OF $\pi_{\text {OTP }}$}

The attack of the adversary is supposed to be divided by two stages. In the first stage, named learning stage, the adversary eavesdrops on the communication channel between the reader and the tag. It gathers not only the all messages transmitted through the channel but also the result, success or fail, of the authentication. In the second stage, named attacking stage, the adversary launches attacks to the RFID system.

The adversary may gather some groups of information $h_{i}\left(\alpha_{i}\right)=\beta_{i}$ transmitted by the tag to the reader during the first attack stage. Later, in the second attack stage, the adversary forges a message $C$ ' and sends it to the reader in the step 2 when the reader authenticating the tag. The random number $\alpha_{q+1}$ can be obtained by the adversary by eavesdropping. But the hash function $h_{i}$ is kept secret to the adversary. The anti-collision characteristic of the hash function ensures the success probability of the adversary is no more than random guessing.

Because the information transferred between the tag and the reader are universally hashed by $h_{i}$, so the situation of privacy and correctness is the same with the security.

\section{CONCLUSIONS}

The ownership transfer protocol $\pi_{\text {OTP }}$ adopts challengeresponse mechanism to realize the mutual authentication of the tag and the reader. The tag replies the query of the reader by pseudorandom number generated by PRNG. The key shared by the tag and the reader is changed by universal hash function after successful authentication. The anticollision characteristic of the hash function provides security, privacy and correctness of the RFID system.
Adopting universal hash function and pseudorandom number generator as security mechanism, the $\pi_{\text {OTP }}$ is a kind of lightweight security and privacy protocol that suits low cost RFID tag such as EPCglobal C1G2.

\section{ACKNOWLEDGEMENTS}

The research work was supported by Science and Technology Development Plan Project of Shandong Province under Grant No. 2013YD01038.

\section{REFERENCES}

[1] J. Saito, K. Imamoto, and K. Sakurai. Reassignment scheme of an RFID tags key for owner transfer[J]. Embedded and Ubiquitous Computing, 2005.p.1303:1312.

[2] M. Ohkubo, K. Suzuki, and S. Kinoshita. Cryptographic Approach to Privacy-Friendly Tags[C]. In RFID Privacy Workshop, 2003.

[3] Moriyama D, Ohkubo M, Matsuo S. A forward privacy model for rfid authentication protocols [M]//nformation Security Theory and Practice. Security of Mobile and Cyber-Physical Systems. Springer Berlin Heidelberg, 2013: 98-111.

[4] Kardaş S, Çelik S, Sariyüce M, et al. An Efficient and Private Authentication Protocol for RFID Systems[J]. Journal of Communications Software \& Systems, 2013, 9(2).

[5] D. Molnar, A. Soppera, D. Wagner, A scalable, delegatable pseudonym protocol enabling ownership transfer of RFID tags[J], Lecture Notes in Computer Science, 2006. vol. 3897, p. 276:290.

[6] C.H. Lim and T. Kwon. Strong and robust rfid authentication enabling perfect ownership transfer[C]. In Proceedings of Conference on Information and Communications Security, p.1:20, Raleigh, USA, 2006.

[7] Berbain C, Billet O, Etrog J, Gilbert H. An efficient forward private RFID protocol[C], Proceedings of the 16th ACM conference on computer and communications Security (ACM CCS'09). Chicago ,USA, 2009:p.43-53.

[8] Kfir, Z., Wool, A..Picking Virtual Pockets using Relay Attacks on Contactless Smartcard Systems[C]. In The First International Conference on Security and Privacy for Emerging Areas in Communications Networks-SecureComm 2005. p.47:58. 